\title{
Ultrafast Charge Carrier Relaxation in Inorganic Halide Perovskite Single Crystals Probed by Two- dimensional Electronic Spectroscopy
}

\author{
Xuan Trung Nguyen ${ }^{1}$, Daniel Timmer' ${ }^{1}$ Yevgeny Rakita ${ }^{2}$,David Cahen ${ }^{2}$, \\ Alexander Steinhoff ${ }^{3}$, Frank Jahnke ${ }^{3}$, Christoph Lienau ${ }^{1}$, Antonietta De Sio ${ }^{1}$ \\ 1 Institut für Physik, Carl von Ossietzky Universität, 26129 Oldenburg, Germany \\ 2 Department of Materials \& Interfaces, Weizmann Institute of Science, Israel \\ 3 Institut für Theoretische Physik, Universität Bremen, Germany \\ Correspondence to: antonietta.de.sio@uni-oldenburg.de
}

\section{Content}

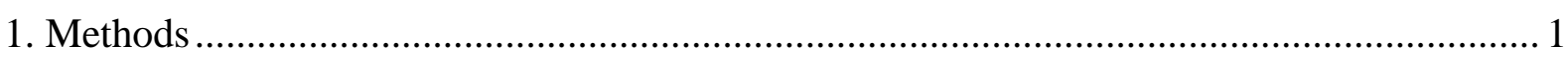

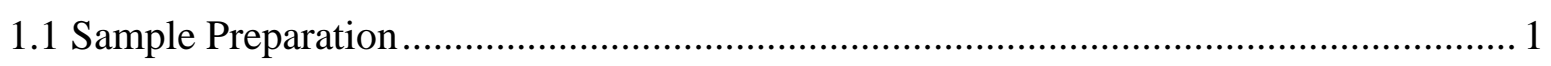

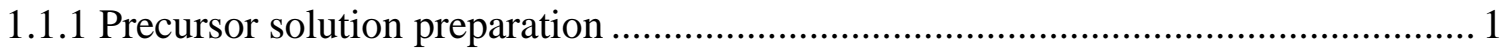

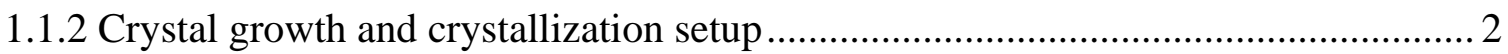

1.2 Pump-probe and two-dimensional electronic spectroscopy (2DES) setup .................... 2

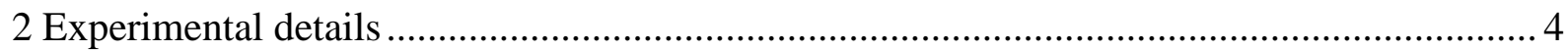

2.1 Temperature dependence of the linear reflectivity .................................................... 4

2.2 Excitation-energy dependence of the experimental differential reflectivity ................... 5

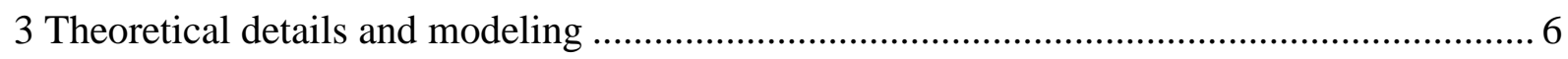

3.1 Semiconductor Bloch equations incl. carrier-carrier and carrier-phonon interaction ...... 6

3.2 Many-body description of excitation-dependent optical spectra................................... 9

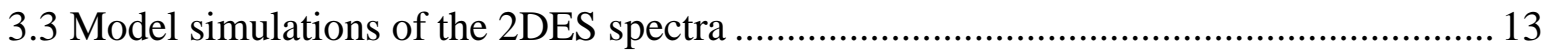

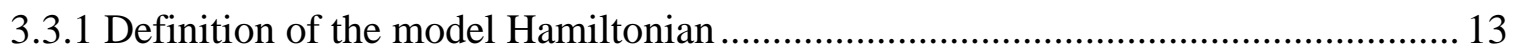

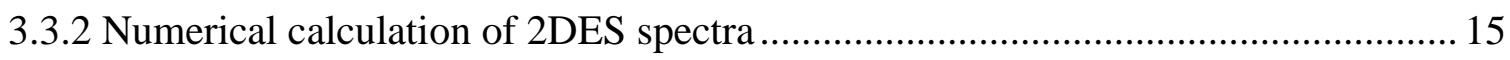

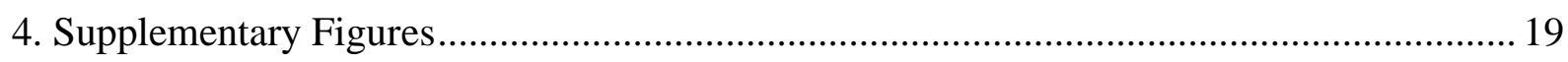




\section{Methods}

\subsection{Sample Preparation}

Powders of cesium bromide (CsBr) (Aldrich, 99.999\%) without further purification and lead bromide $(\mathrm{PbBr} 2)$ (Aldrich, $\geq 98 \%$ ) that was dried in a vacuum oven overnight were dissolved in dimethylsulfoxide (DMSO) (Aldrich, $\geq 99.9 \%$ ). Acetonitrile (MeCN) (BioLab LTD, HPLC grade, 99.97\%) and methanol (MeOH) (BioLab LTD, HPLC grade, 99.95\%) were used as received.

\subsubsection{Precursor solution preparation}

A $0.45 \mathrm{M}$ solution (slightly below the $\sim 0.5 \mathrm{M}$ solubility limit) of the perovskite precursors (equimolar amounts of $\mathrm{CsBr}$ and $\mathrm{PbBr}_{2}$ ) in the same solution of DMSO was prepared in ambient air (ca. $45 \% \mathrm{RH})$ under continuous stirring at $\sim 50^{\circ} \mathrm{C}$, until no powder is observed. It is important to mix the precursors in the same volume, because the room temperature solubility limit of $\mathrm{CsBr}$ in DMSO is ca. 0.25 M when it is dissolved separately. After cooling to room temperature, the DMSO solution was titrated (dropwise under continuous stirring) with MeCN. During the titration, a yellow-orange precipitant appeared with addition of each drop and quickly redissolved. As the system gets closer to the saturation point (more pronounced when $\mathrm{MeCN}$ is added), a permanent white solid precipitates. At MeCN : DMSO ratios of $1.1: 1$, the yelloworange precipitate no longer re-dissolved. These saturated solutions were thoroughly sealed (to prevent loss of the volatile $\mathrm{MeCN}$ ) and stirred for 24 hours at $50{ }^{\circ} \mathrm{C}$. After heating for $24 \mathrm{~h}$, a pale green-yellow strongly-fluorescent precipitant was clearly observed along-side the other precipitated species. The saturated solutions can be further stored (best in the dark) for at least several months, until their use for crystal growth. Before crystal growth, the saturated precursor solutions are filtered with PTFE $0.2 \mu \mathrm{m}$ pore-size syringe filters. No noticeable differences in crystal growth or kinetics were observed between properly stored or freshly prepared solutions. 


\subsubsection{Crystal growth and crystallization setup}

A scheme of the crystallization setup is presented in Figure S1a. The filtered precursor solutions were placed in a clean crystallization flask and covered with a filter paper and a glass petri-dish on top to limit anti-solvent vapor diffusion. The covered crystallization flask was then placed inside a deeper, flat-bottomed, glass dish, which contained $\mathrm{MeCN}$ (anti-solvent). The setup was left at ambient conditions in a quiet location for further growth. After approximately 2-3 days the crystals were taken out (Figure S1b). Typical single crystals collected from the crystallization solution are depicted in Figure S1b-c. The crystallographic orientation is verified via XRD analysis in a specular reflection mode, where the (101) plane is set as the reflection plane (Figure S1c). The most common crystal geometry was rectangular with (101) as most pronounced facet (Figure S1c). For further details see Ref. 1.

\subsection{Pump-probe and two-dimensional electronic spectroscopy (2DES) setup}

The home-built experimental setup used to record pump-probe and 2DES spectra in reflection geometry is schematically depicted in Figure S2a. A home-built non-collinear optical parametric amplifier (NOPA), pumped by the second harmonic of a regeneratively amplified Ti:Sapphire laser (Spectra Physics Spitfire Pro, 150 fs pulses centered at $800 \mathrm{~nm}, 5 \mathrm{kHz}$ repetition rate), is used to generate ultrabroadband pulses with a spectrum ranging from $\sim 2.1 \mathrm{eV}$ to $\sim 2.6$ eV. The pulses are compressed with chirped mirrors (Laser Quantum DCM9). Pump-probe and 2DES maps are recorded in a partially collinear geometry (Figure S2a). The passively phaselocked pump pulse-pair is generated by means of a common-path delay line based on $\alpha$-BBO wedges (TWINS). ${ }^{2}$ An additional pair of chirped mirrors is used in the pump arm to compensate the dispersion introduced by the TWINS. Motorized delay stages (Physik Instrumente M112.1DG and M413.2DG) are used to scan the time delay between the two pump pulses, $\tau$ (coherence time), and between the pump and probe arm, $T$ (waiting time). A scheme of the pulse sequence is reported in Figure $S 2 b$. To calibrate the coherence time $\tau$, a small fraction of 
the pump beam is sent to a photodiode that records the autocorrelation of the two pump pulses. ${ }^{3}$ For the pump-probe measurements, $\tau$ is set to zero. Pump and probe beams are focused on the sample to a spotsize of $\sim 80 \mu \mathrm{m}$. The exact beam area is carefully characterized by a beam camera at the focus position, allowing for an accurate determination of the fluence. The polarization angle between the linearly polarized pump and probe pulses is tuned to $\sim 55^{\circ}$. The reflected probe beam from the sample is dispersed in a monochromator and recorded with a 1024pixel CCD-array (Entwicklungsbüro Stresing), whereas the reflected pump beam is blocked by an iris.

The signal $R\left(E_{D}\right)$ at detection energy $E_{D}$ recorded by the CCD detector is proportional to the amplitude squared of the interference between the probe pulse $\tilde{E}_{R}\left(E_{D}\right)$, reflected from the surface and used as the reference pulse, and the signal field $\tilde{E}_{S}\left(E_{D}\right)$, emitted by the nonlinear polarization of the sample,

$$
R\left(E_{D}\right)=\left|\tilde{E}_{R}\left(E_{D}\right)+\tilde{E}_{S}\left(E_{D}\right)\right|^{2}
$$

Here, $\tilde{E}_{R, S}\left(E_{D}\right)=E_{R, S}\left(E_{D}\right) e^{i \varphi_{R, S}\left(E_{D}\right)}$ are the Fourier transforms of the time-domain reference (R) and signal (S) fields, respectively. Their real amplitudes are denoted as $E_{R, S}\left(E_{D}\right)$ and their spectral phases as $\varphi_{R, S}\left(E_{D}\right)$. We can write the signal field as $\tilde{E}_{S}=\tilde{r}_{N L} \cdot \tilde{E}_{R}=r_{N L} \cdot E_{R} \cdot e^{i\left(\varphi_{R}+\varphi_{r}\right)}$, with $\tilde{r}_{N L}=r_{N L} e^{i \varphi_{r}}$ denoting the nonlinear optical response function of the sample. The signal at the detector then is

$$
R\left(\omega_{D}\right)=E_{R}^{2}\left(1+r_{N L}^{2}+2 r_{N L} \cos \varphi_{r}\right)=E_{R}^{2}\left(1+r_{N L}^{2}+2 \operatorname{Re}\left(\tilde{r}_{N L}\right)\right)
$$

The first term is suppressed by modulating the pump pulses with a mechanical chopper at a frequency of $500 \mathrm{~Hz}$. The second term is small and can generally be neglected. This shows that, in our experimental configuration, both pump-probe $\Delta R / R$ and 2DES signals measure the real 
part of $\tilde{E}_{S}$ with respect to the phase of $\tilde{E}_{R}$. In a transmission geometry this gives rise to absorptive bleaching peaks in pump-probe and 2DES spectra. ${ }^{4-5}$ The $90^{\circ}$ phase shift acquired by the probe pulses upon reflection at the sample surface results in dispersive line shapes in reflection geometry. ${ }^{6}$

The measured signal is the differential reflection from the sample $\frac{\Delta R}{R}\left(\tau, T, E_{D}\right)=\frac{R_{o n}\left(\tau, T, E_{D}\right)-R_{o f f}\left(E_{D}\right)}{R_{o f f}\left(E_{D}\right)}$ as a function of $\tau$ and $T$ and of the detection energy $E_{D}$. Here, $R_{o n}$ and $R_{\text {off }}$ denote the reflected probe laser spectra with the pump being switched on and off, respectively. The pump beam is modulated with a mechanical chopper at a frequency of $500 \mathrm{~Hz}$. Absorptive 2DES maps $A_{2 D}\left(E_{X}, T, E_{D}\right)$ are obtained by taking the real part of the Fourier transform of the measured $\Delta R / R$ signal along $\tau$ to obtain the excitation energy axis $E_{X}$, $A_{2 D}\left(E_{X}, T, E_{D}\right)=\operatorname{Re}\left(\int_{0}^{\infty} \frac{\Delta R}{R}\left(\tau, T, E_{D}\right) \exp \left(i E_{D} \tau / \hbar\right) d \tau\right)$. A Gaussian filter $G(\tau)=e^{-4 \ln 2\left(\frac{\tau}{\tau_{F}}\right)^{2}}$, with full width at half maximum $\tau_{F}=180 \mathrm{fs}$, is used to minimize the effect of truncation on the Fourier transform. To characterize the time resolution of the nonlinear experiments, we record second-harmonic generation frequency-resolved optical gating (SHG-FROG) ${ }^{7}$ maps generated by the cross-correlation between pump and probe beams at the sample position. Figure S2c reports a typical SHG-FROG map from which we estimate a time resolution of $<13$ fs.

\section{Experimental details}

\subsection{Temperature dependence of the linear reflectivity}

The absorption spectrum of our $\mathrm{CsPbr}_{3}$ samples shows a saturation plateau for energies $>2.3$ $\mathrm{eV}$ (Figure 1a, blue in the manuscript). This saturation results from the large thickness of the crystals, far exceeding the penetration depth for near-bandgap photon energies. This makes it 
impossible to probe signatures of near-band-edge absorption in transmission measurements. For this reason, all optical measurements are performed in reflection geometry.

The linear reflectivity of the $\mathrm{CsPbBr}_{3}$ crystals at room temperature shows a broadband background reflectivity of $6-9 \%$ and a dispersive near-band-edge reflectivity peak at $\sim 2.4 \mathrm{eV}$, reflecting both excitonic and free carrier transitions close to the band-edge (Figure S3, pink). The maximum amplitude of this dispersive peak is about $1.5 \%$. In these linear optical spectra, excitonic and free-carrier contributions to the reflectivity peak cannot be distinguished. Therefore, we record the temperature dependence of the linear reflectivity for our $\mathrm{CsPbBr}_{3}$ single crystals. The sample is fixed on the cold finger of a liquid nitrogen cryostat and the reflectivity is recorded from $292 \mathrm{~K}$ down to $65 \mathrm{~K}$ in about $20 \mathrm{~K}$ steps (Figure S3). With decreasing temperature, we observe a narrowing of the near-band-edge reflectivity peak and a monotonic redshift of its resonance energy from $2.4 \mathrm{eV}$ at room temperature to $\sim 2.36 \mathrm{eV}$ at $65 \mathrm{~K}$, in good agreement with previous reports in the literature.$^{8-9}$ Even at low temperatures, however, the linear reflectivity shows essentially a single dispersive resonance (Figure S3), and excitonic and free carrier contributions to the spectrum cannot be separated.

\subsection{Excitation-energy dependence of the experimental differential reflectivity}

To study the effect of the excitation fluence on the nonlinear optical spectra of $\mathrm{CsPbBr}_{3}$ single crystals, we recorded differential reflectivity $\Delta R / R$ spectra at a series of different pump fluences, increasing the pump pulse energy from $2 \mathrm{~nJ}$ to $14 \mathrm{~nJ}$. The corresponding fluence was calculated after characterization of the beam spot size at the focus position with a beam-camera (Thorlabs DCC1645C CMOS Camera, 1280 x 1024, Color Sensor). For the pump beam, the spotsize at the focus is $94 \times 82 \mu \mathrm{m}^{2}$, resulting in fluences ranging from $\sim 8 \mu \mathrm{J} / \mathrm{cm}^{2}$ to $\sim 230$ $\mu \mathrm{J} / \mathrm{cm}^{2}$. The probe is focused to a slightly smaller spot size of $83 \times 54 \mu \mathrm{m}$, delivering a fluence of $28 \mu \mathrm{J} / \mathrm{cm}^{2}$. In all experiments, the probe pulse fluence was kept constant. Figure S4 shows the differential reflectivity as a function of pump pulse energy. The $\Delta R / R$ dynamics (Figure 
$\mathrm{S} 4 \mathrm{~b}, \mathrm{c}$ ) show essentially no variation for time delays up to $\sim 180 \mathrm{fs}$, i.e., on the timescale relevant for the nonlinear measurements presented in the manuscript. We observe a linear correlation between pump pulse energy and $\Delta R / R$ signal for the measured energy range (Figure $\mathrm{S} 4 \mathrm{~d}$ ). No signs of sample degradation during the entire measurement process are observed. All the data presented in the manuscript are recorded at a pump fluence of $90 \mu \mathrm{J} / \mathrm{cm}^{2}$.

To estimate the photogenerated carrier densities, we consider an absorption coefficient of $\alpha \sim 2 \cdot 10^{4} \mathrm{~cm}^{-1}$ at photon energies of $>2.3 \mathrm{eV}$, in agreement with previous reports from some of us ${ }^{1}$ and from other groups ${ }^{10-11}$. This value results in a penetration depth of $\sim 500 \mathrm{~nm}^{1}$. Together with the measured pump fluence, we obtain carrier densities ranging from $\sim 4 \cdot 10^{17} \mathrm{~cm}^{-3}$ to $\sim 1 \cdot 10^{19} \mathrm{~cm}^{-3}$ for the excitation conditions reported in Figure S4. For both differential reflectivity and 2DES data analyzed in the main manuscript, the estimated density of photogenerated carriers is $\sim 4.6 \cdot 10^{18} \mathrm{~cm}^{-3}$. This is similar to the carrier densities used in the 2DES studies of methylammonium lead iodide thin films reported in Refs. ${ }^{11-12}$. A comparison with the optical spectra deduced from semiconductor Bloch equations shows however that this value overestimates the actual excited carrier density by approximately an order of magnitude. This is discussed in section 3.2 .

\section{Theoretical details and modeling}

\subsection{Semiconductor Bloch equations including carrier-carrier and carrier-phonon interaction}

The absorption of a semiconductor medium is obtained from its linear response to a classical electric field $E(t) .{ }^{13}$ The electric field drives a macroscopic polarization of the medium that is composed of the microscopic inter-band polarizations $\psi_{k}^{h e}(t)=<a_{k}^{h} a_{k}^{e}>(t)$, with the annihilation operator $a_{k}^{h / e}$ of electrons and holes, according to $P(t)=\frac{1}{V} \sum_{k, e h} \psi_{k}^{h e}(t)\left(d_{k}^{\text {eh }}\right)^{*}+$ c.c. 
Here, $V$ is the crystal volume and $d_{k}^{e h}$ are dipole matrix elements describing the efficiency of light-matter coupling depending on the momentum $k$. In the regime of weak excitation, the macroscopic polarization is linear in the electric field. This allows for extracting the response of the medium as a pure material property in the form of a susceptibility that is independent of the electric field: $\chi(\omega)=\frac{P(\omega)}{\varepsilon_{0} E(\omega)}$, where we consider an isotropic susceptibility $\chi(\omega)$. To obtain the frequency-dependent response of the medium, we use the Fourier transform of the macroscopic polarization $P(t)$, assuming the medium to be in a quasi-equilibrium state. In this picture, any excited carriers that are generated by pumping the active medium relax to quasiequilibrium distributions at a certain temperature and carrier density. The absorption at these parameters is then probed by the weak electric test field.

Applying the technique of nonequilibrium Green functions, the equation of motion for microscopic inter-band polarizations, known as semiconductor Bloch equations (SBE), can be derived. ${ }^{14-15}$ In the following, we use the SBE formulated in frequency space taking into account many-body effects due to excited carriers on a GW-level. ${ }^{16}$ Furthermore, carrier-phonon interaction in random phase approximation (RPA) can be introduced in the same spirit, leading to the equation of motion for $\psi_{k}^{h e}(\omega)$ :

$$
\begin{aligned}
& \left(\hbar \omega-\varepsilon_{k}^{H F, e}-\varepsilon_{k}^{H F, h}-\Delta_{k}^{e h, \text { Coul }}(\omega)-\Delta_{k}^{\text {eh,carr-phon }}\right) \psi_{k}^{\text {he }}(\omega) \\
& +\left(1-f_{k}^{h}-f_{k}^{e}\right)\left(d_{k}^{e h} E(\omega)+\frac{1}{V} \sum_{k^{\prime}} V_{k-k^{\prime}} \psi_{k^{\prime}}^{h e}(\omega)\right) \\
& +\frac{1}{V} \sum_{k^{\prime}}\left(V_{k, k^{\prime}}^{\text {eff eh,Coul }}(\omega)+V_{k, k^{\prime}}^{\text {eff, eh,carr-phon }}\right) \psi_{k^{\prime}}^{\text {he }}(\omega)=0
\end{aligned}
$$

Just as the well-known Bethe-Salpeter equation in screened ladder approximation, ${ }^{17-18}$ the SBE including carrier-carrier interaction on the GW-level describe two-particle states in the presence of a dynamically screened carrier-carrier interaction. Carrier-phonon interaction leads to additional polaron effects. The single-particle energies $\varepsilon_{k}^{H F, e / h}$ include renormalization effects on 
a Hartree-Fock level. The second line contains the light-matter coupling term $d_{k}^{e h} E(\omega)$ of the materials' dipoles to the external field as well as a two-body interaction term facilitating excitonic resonances in the optical response. These terms are modified by a Pauli-blocking factor given by the electron and hole occupancies for which we assume Fermi distribution functions.

All many-body effects induced by carrier-carrier interaction beyond the Hartree-Fock level are contained in the correlation terms

$$
V_{k, k^{\prime}}^{\text {eff,eh,Coul }}(\omega)=i \hbar \int_{-\infty}^{\infty} \frac{d \omega^{\prime}}{2 \pi}\left\{\frac{\left(1-f_{k}^{h}+n_{B}\left(\omega^{\prime}\right)\right) 2 i \operatorname{Im} W_{k-k^{\prime}}^{r e t}\left(\omega^{\prime}\right)}{\hbar \omega-\varepsilon_{k}^{h}-\varepsilon_{k^{\prime}}^{e}+i \Gamma_{k}^{h}+i \Gamma_{k^{\prime}}^{e}-\hbar \omega^{\prime}}+\frac{\left(1-f_{k}^{e}+n_{B}\left(\omega^{\prime}\right)\right) 2 i \operatorname{Im} W_{k-k^{\prime}}^{r e t}\left(\omega^{\prime}\right)}{\hbar \omega-\varepsilon_{k}^{e}-\varepsilon_{k^{\prime}}^{h}+i \Gamma_{k}^{e}+i \Gamma_{k^{\prime}}^{h}-\hbar \omega^{\prime}}\right\}
$$

with the Bose distribution function $n_{B}(\omega)$ and Fermi distribution function $f_{k}^{e / h}$, and

$$
\Delta_{k}^{e h, \text { Coul }}(\omega)=\Sigma_{k}^{M W, r e t, e}\left(\hbar \omega-\varepsilon_{k}^{h}+i \Gamma_{k}^{h}\right)+\Sigma_{k}^{M W, r e t, h}\left(\hbar \omega-\varepsilon_{k}^{e}+i \Gamma_{k}^{e}\right)
$$

where the Montroll-Ward (MW) self-energy

$$
\Sigma_{k}^{M W, r e t, a}(\omega)=i \hbar \int_{-\infty}^{\infty} \frac{d \omega^{\prime}}{2 \pi} \frac{1}{V} \sum_{k^{\prime}} \frac{\left(1-f_{k^{\prime}}^{a}+n_{B}\left(\omega^{\prime}\right)\right) 2 i \operatorname{Im} W_{k-k^{\prime}}^{r e t}\left(\omega^{\prime}\right)}{\hbar \omega-\varepsilon_{k^{\prime}}^{a}+i \Gamma_{k^{\prime}}^{a}-\hbar \omega^{\prime}}
$$

is responsible for quasi-particle renormalizations beyond Hartree-Fock according to

$$
\varepsilon_{k}^{a}=\varepsilon_{k}^{0, a}+\Sigma_{k}^{H F, a}+\operatorname{Re} \Sigma_{k}^{M W, r e t, a}\left(\varepsilon_{k}^{a} / \hbar\right)+\operatorname{Re} \sum_{k}^{\text {carr }- \text { phon, }, \text { ret, }, a}\left(\varepsilon_{k}^{a} / \hbar\right) .
$$

The corresponding quasi-particle broadening follows from the imaginary part of the MW selfenergy:

$$
\Gamma_{k}^{a}=-\operatorname{Im} \sum_{k}^{M W, r e t, a}\left(\varepsilon_{k}^{a} / \hbar\right)-\operatorname{Im} \sum_{k}^{\text {carr }- \text { phon, }, \text { ret }, a}\left(\varepsilon_{k}^{a} / \hbar\right) .
$$

Since the focus of this work is on excitation-induced dephasing, we keep the description of carrier-phonon interaction as simple as possible using a Markov approximation, thereby neglecting the frequency-dependence of dephasing. To evaluate the carrier-phonon correlations, we take into account renormalized single-particle energies, which are supplemented by a fixed quasi-particle relaxation rate of $\Gamma_{0}=20 \mathrm{meV}$ corresponding to typical carrier-phonon scattering 
times. ${ }^{19}$ Due to the fixed quasi-particle decay rates, contributions from different phonon branches $j$ are practically decoupled. Then the correlation contributions to the SBE are given by

$$
V_{k, k^{\prime}}^{\text {eff,eh,carr-phon }}=\sum_{j}\left\{\frac{\left(1-f_{k}^{h}+n_{B}\left(\Omega_{k-k^{\prime}}^{j}\right)\right) g_{k-k^{\prime}}^{e . j}\left(g_{k-k^{\prime}}^{h}\right)^{*}}{\varepsilon_{k}^{e}-\varepsilon_{k^{\prime}}^{e}+2 i \Gamma_{0}-\hbar \Omega_{k-k^{\prime}}^{j}}+\frac{\left(f_{k}^{h}+n_{B}\left(\Omega_{k-k^{\prime}}^{j}\right)\right) g_{k-k^{\prime}}^{e . j}\left(g_{k-k^{\prime}}^{h}\right)^{*}}{\varepsilon_{k}^{e}-\varepsilon_{k^{\prime}}^{e}+2 i \Gamma_{0}+\hbar \Omega_{k-k^{\prime}}^{j}}+(e \leftrightarrow h)\right\}
$$

and

$$
\Delta_{k}^{e h, e l-p h}=\Sigma_{k}^{e l-p h, r e t, e}\left(\varepsilon_{k}^{e}+i \Gamma_{0}\right)+\Sigma_{k}^{e l-p h, r e t, h}\left(\varepsilon_{k}^{h}+i \Gamma_{0}\right)
$$

with the self-energy

$$
\sum_{k}^{e l-p h, r e t, a}(\omega)=\frac{1}{V} \sum_{k^{\prime}, j}\left\{\frac{\left(1-f_{k^{\prime}}^{a}+n_{B}\left(\Omega_{k-k^{\prime}}^{j}\right)\right)\left|g_{k-k^{\prime}}^{a . j}\right|^{2}}{\hbar \omega-\varepsilon_{k^{\prime}}^{a}+i \Gamma_{0}-\hbar \Omega_{k-k^{\prime}}^{j}}+\frac{\left(f_{k^{\prime}}^{a}+n_{B}\left(\Omega_{k-k^{\prime}}^{j}\right)\right)\left|g_{k-k^{\prime}}^{a . j}\right|^{2}}{\hbar \omega-\varepsilon_{k^{\prime}}^{a}+i \Gamma_{0}+\hbar \Omega_{k-k^{\prime}}^{j}}\right\} .
$$

We use long-wavelength phonon frequencies $\Omega_{q}^{j}$ and carrier-phonon matrix elements $g_{q}^{a, j}$ describing the Fröhlich-type interaction with longitudinal-optical phonons as given in Ref. ${ }^{19}$.

For the band-structure energies $\varepsilon_{k}^{0}$ in the absence of excitation-induced renormalizations, we apply an effective mass approximation with parameters given above. The correlation terms contain the retarded screened potential $W_{k-k^{\prime}}^{\text {ret }}(\omega)=V_{k-k^{\prime}} \varepsilon_{k-k^{\prime}}^{-1, e x c}(\omega)$, where the inverse dielectric function $\varepsilon_{q}^{-1, e x c}(\omega)$ describes screening due to excited carriers in random phase approximation (RPA). Additionally, $V_{q}$ is screened by carriers in filled valence-band states, which we describe by a dielectric constant. The SBE are numerically solved by matrix inversion for each frequency to obtain the frequency-dependent microscopic polarization and thereby the optical susceptibility $\chi(\omega)$.

\subsection{Many-body description of excitation-dependent optical spectra}

Insight into the underlying contributions to the excitation-dependent changes of optical properties is gained by solving the SBE to describe excitonic and free-carrier contributions to the 
optical susceptibility $\chi(\omega)$ near the fundamental band gap. A detailed presentation of the SBE is given in section 3.1. The SBE contain many-body effects due to excited carriers in the form of Pauli-blocking, screening of the Coulomb interaction, energy renormalization (including band-gap shrinkage), and excitation-induced dephasing. In the present calculation, many-particle effects of excited carriers are included in GW-approximation for the Coulomb self-energy contributions to the SBE. Additionally, we include effects of carrier-phonon interaction in terms of the RPA self-energy describing Fröhlich-type coupling to longitudinal-optical phonons. The coupling strength and long-wavelength phonon frequencies are parametrized based on density functional theory results of Ref. ${ }^{19}$. Using first-principle-based k-dot-p parameters from Ref. ${ }^{20}$, we assume parabolic bands near the fundamental band gap with $E_{g a p}=2.36 \mathrm{eV}$ at the R-point of the Brillouin zone and effective masses of $m_{e}=0.134 m_{0}$ and $m_{h}=0.128 m_{0}$, for electrons and holes, respectively. Momentum-independent dipole matrix elements $d_{c v}=0.52 \mathrm{~nm}$ are obtained from the corresponding $E_{P}$ parameter. For the Coulomb matrix elements, we use a $1 / q^{2}$ dependency with a high-frequency dielectric background screening of $\varepsilon_{\infty}=4.8$ taken from the same reference. ${ }^{20}$ These parameters result in a three-dimensional exciton binding energy of 38.7 meV.

The linear absorption spectra for different carrier densities $\mathrm{n}$ are shown in Figure S5a. Here a quasi-equilibrium distribution of excited carriers has been assumed for a temperature of $300 \mathrm{~K}$. For the lowest carrier density of $\mathrm{n}=1 \cdot 10^{16} \mathrm{~cm}^{-3}$ (Figure S5 orange), a pronounced exciton resonance is obtained around the photon energy of $2.275 \mathrm{eV}$. Efficient carrier-phonon interaction results in substantial line broadening and a polaron shift of the order of $50 \mathrm{meV}$. Due to the strong broadening, higher excitonic states and the Coulomb-interaction enhanced band-to-band transitions merge into a nearly flat absorption region above the excitonic ground-state transition. 
With increasing carrier density, the bleaching of the excitonic transitions occurs due to Pauliblocking as well as due to screening of the Coulomb interaction, which is included in our calculation in dynamical random-phase-approximation. At the same time, the band gap shifts to lower energies, thereby red-shifting regions of higher density-of-states to energies between 2.3 and $2.4 \mathrm{eV}$. The interplay of these effects explains the decreasing (increasing) absorption below (above) $2.3 \mathrm{eV}$ with increasing carrier density (Figure S5a). At elevated carrier densities, Coulomb interaction causes additional strong linewidth broadening. The excitation-induced linewidth broadening, which is obtained from microscopic calculations of the Coulomb interaction and carrier-phonon interaction, can be extracted by fitting a Sech function to the low-energy side of the exciton resonance in Figure S5a. The resulting values of the half-width-at-half-maximum (HWHM) are given in Table 1. At carrier densities close to $1 \cdot 10^{18} \mathrm{~cm}^{-3}$, the sharp excitonic resonance around $2.275 \mathrm{eV}$ disappears. This is the distinct signature of reaching the excitonic Mott transition.

\begin{tabular}{|l|l|l|l|l|}
\hline $\mathrm{n}\left(\mathrm{cm}^{-3}\right)$ & $1 \cdot 10^{15}$ & $1 \cdot 10^{16}$ & $1 \cdot 10^{17}$ & $3 \cdot 10^{17}$ \\
\hline HWHM (meV) & 21 & 21 & 23 & 27 \\
\hline
\end{tabular}

Table 1: Linewidth (HWHM) of excitonic resonance depending on excited carrier density.

In general, in order to obtain reflectivity spectra $R(\omega)$, a self-consistent solution of the SBE together with Maxwell's equations for the propagating light field is necessary. For thick bulk crystals, $R(\omega)$ can be approximated using Fresnel equations and a complex refractive index $n(\omega)=\sqrt{\varepsilon(\omega)}=\sqrt{n_{B G}^{2}+\chi(\omega) / \varepsilon_{0}}$ with $\chi(\omega)$ following from a numerical solution of the SBE. Reflectivity spectra calculated in this way as a function of excited carrier density are shown in Figure S5b. Since the reflectivity is dominated by $\operatorname{Re} \chi(\omega)$ and the real and imaginary parts of the susceptibility are related via a Kramers-Kronig transform, the peak positions in $R(\omega)$ do not directly coincide with the exciton resonance. Figure S5b shows that the sharp dispersive 
resonant feature in the linear reflectivity around $2.275 \mathrm{eV}$ arises from excitonic and Coulombenhanced free carrier transitions near the band-edge. Its amplitude tends to decrease with increasing excitation density. Near the Mott transition, around $10^{18} \mathrm{~cm}^{-3}$, it vanishes and a much broader positive peak remains (red line in Figure S5b). The calculated nonlinear, differential reflectivity spectra $\Delta R / R$ for different carrier densities $\mathrm{n}$ are provided in Figure 1e of the manuscript. They show a dispersive feature centered around $2.275 \mathrm{eV}$ with a shape that is very similar to that seen experimentally. It is the consequence of the excitation-induced bleaching of excitonic transitions due to Pauli-blocking and screening of Coulomb interactions and the effect of the nonequilibrium charge carriers on the free-carrier transitions near the band edge. For sufficiently low excitation densities its amplitude increases linearly with density, before it saturates and reaches a maximum value of $\Delta R / R$ of $\sim 8 \%$ for an excitation density of $10^{18} \mathrm{~cm}^{-3}$, close to the Mott transition. This maximum amplitude nicely matches that seen experimentally in Figure S4d. This directly shows that the experiments in Figures 1-3 of the manuscript, recorded at a pump fluence of $90 \mu \mathrm{J} / \mathrm{cm}^{2}$ and displaying maximum $\Delta R / R$ amplitudes of $2 \%$, are performed at an excitation density well below the Mott transition. From Figure 1e of the manuscript, we estimate an excitation density of $\sim 3 \cdot 10^{17} \mathrm{~cm}^{-3}$.

A striking observation is the almost perfectly stable zero crossing of the differential shape for different carrier densities up to about $6 \cdot 10^{17} \mathrm{~cm}^{-3}$ (Figure 1e, dark-grey), whereas a slight blueshift is observed at higher densities. The amplitude of this peak thus provides a measure for the density of nonequilibrium charge carriers in the system. The long-time decay of this amplitude that is seen in Figure 1d thus can be traced back to the onset of carrier recombination and to the transport of charge carriers out of the sample volume that is seen by the probe laser. 


\subsection{Model simulations of the 2DES spectra}

\subsubsection{Definition of the model Hamiltonian}

Given the numerical complexity involved in solving the complete semiconductor Bloch equations model, we use a simpler, phenomenological optical Bloch equation model to simulate the 2DES spectra shown in the main manuscript (Figure 4a, b). For this, we describe both the inhomogeneously broadened exciton (X) and the free carrier continuum (C) phenomenologically as a series of uncoupled, i.e., non-interacting, two-level systems (TLSs). The Hamiltonian of each TLS is defined as $H_{0, k}=\hbar \omega_{0, k}\left|0_{k}\right\rangle\left\langle 0_{k}\left|+\hbar \omega_{1, k}\right| 1_{k}\right\rangle\left\langle 1_{k}\right|$. Here $\left|0_{k}\right\rangle$ and $\left|1_{k}\right\rangle$ denote the electronic ground and excited states of the $k$-th TLS, and $\hbar \omega_{0, k}$ and $\hbar \omega_{1, k}$ are their associated eigenenergies. For simplicity, in our simulations we set the ground state energy $\hbar \omega_{0, k}=0 \mathrm{eV}$ for each TLS,

$$
H_{0, k}=\hbar \omega_{1, k}\left|1_{k}\right\rangle\left\langle 1_{k}\right|
$$

Interaction with light, inducing transitions between $\left|0_{k}\right\rangle$ and $\left|1_{k}\right\rangle$, is described in point-dipole approximation $^{21}$ as $\hat{H}_{I}(t)=\hat{\mu} \sum_{j} E_{j}(t)$, with the dipole operator $\hat{\mu}$. Each impulsive laser field $E_{j}(t)$ interacting with the system is modeled as a Gaussian pulse with carrier frequency of $2.4 \mathrm{eV}$ and duration of $6 \mathrm{fs}$, defined as the full-width-at-half-maximum (FWHM) of the temporal intensity profile. For the $k$-th TLS, the light-matter interaction part of the Hamiltonian thus reads

$$
H_{I, k}(t)=-\mu_{k} E(t)\left(\left|1_{k}\right\rangle\left\langle 0_{k}|+| 0_{k}\right\rangle\left\langle 1_{k}\right|\right),
$$

where the dipole moment $\mu_{k}=\left\langle 0_{k}|\hat{\mu}| 1_{k}\right\rangle$ is a constant and the operators $\left|1_{k}\right\rangle\left\langle 0_{k}\right|$ and $\left|0_{k}\right\rangle\left\langle 1_{k}\right|$ describe absorption and stimulated emission processes, respectively. 
Since the TLSs do not interact, the global Hamiltonian $H_{0}$, describing the entire system of $N$ uncoupled TLSs, can be written as

$$
H_{0}=\sum_{k=1}^{N} H_{0, k}=\sum_{k=1}^{N}\left(\hbar \omega_{1, k}\left|1_{k}\right\rangle\left\langle 1_{k}\right|\right)
$$

This is formally the same model as employed for e.g., quantum spin chains. ${ }^{22}$ The corresponding light-matter interaction can be written as the sum of the light-matter interactions $H_{I, k}(t)$ of each TLS

$$
H_{I}=\sum_{k=1}^{N} H_{I, k}=-\sum_{k=1}^{N}\left[\mu_{k} E(t)\left(\left|1_{k}\right\rangle\left\langle 0_{k}|+| 0_{k}\right\rangle\left\langle 1_{k}\right|\right)\right]
$$

Specifically, we model the X part of the spectrum (Figure 4a) as a system of $N=3$ uncoupled TLSs with the resonance energy difference between ground and excited states, $\hbar \omega_{x, k}$, with $k=1,2,3$, chosen to simulate an inhomogeneous broadening with FWHM of $\sim 50$ meV around $2.4 \mathrm{eV}$. The transition dipole moment $\mu_{X, k}$ is a constant and is the same for all three TLSs. The free carrier continuum $\mathrm{C}$ (Figure $4 \mathrm{~b}$ ) is modeled as a system of $N=6$ uncoupled TLSs with resonance energies $\hbar \omega_{C, k}$, with $k=1, \ldots, 6$, ranging from $2.43 \mathrm{eV}$ to $2.63 \mathrm{eV}$ and separated by $40 \mathrm{meV}$. The transition dipole moment $\mu_{C, k}$ of each TLS mimicking the continuum is chosen following the Elliot model for free carrier absorption in a three-dimensional semiconductor crystal including Coulomb enhancement. ${ }^{13,23}$ This allows us to obtain realistic absorption and reflectivity spectra of free carriers close to the band-edge. In the Elliot model for the free carrier continuum (C model), the transition dipole moment is defined as

$$
\mu_{C, k}=\alpha_{\mu, k} \sqrt{\Delta_{k}} \Theta\left(\Delta_{k}\right) \frac{\frac{\pi}{\sqrt{\Delta_{k}}} \exp \left(\frac{\pi}{\sqrt{\Delta_{k}}}\right)}{\sinh \left(\frac{\pi}{\sqrt{\Delta_{k}}}\right)},
$$


where $\Delta_{k}=\frac{\hbar \omega_{C, k}-\left(\hbar \omega_{X}+E_{0}\right)}{E_{0}}, \hbar \omega_{X}$ is the exciton resonance energy at $2.4 \mathrm{eV}, E_{0}$ the exciton binding energy, $\alpha_{\mu, k}$ a constant, $\Theta\left(\Delta_{k}\right)$ the Heaviside step function and $C\left(\Delta_{k}\right)=\frac{\frac{\pi}{\sqrt{\Delta_{k}}} \exp \left(\frac{\pi}{\sqrt{\Delta_{k}}}\right)}{\sinh \left(\frac{\pi}{\sqrt{\Delta_{k}}}\right)}$ is the Coulomb enhancement factor. ${ }^{13}$

For the simulations presented in the manuscript (Figure 4), we set the exciton binding energy to $E_{0}=30 \mathrm{meV}$, according to the value experimentally extracted from our room temperature 2DES spectra at $T=0 \mathrm{fs}$ (Figure 3c). This value is also in very good agreement with the one reported in the literature for $\mathrm{CsPBr}_{3}{ }^{9}$

\subsubsection{Numerical calculation of 2DES spectra}

The quantity of interest to calculate optical spectra (both linear and nonlinear) is the expectation value of the dipole operator $\langle\hat{\mu}\rangle$. It contains in fact all relevant information about the optical response of the system upon interaction with one or more optical pulses. A detailed treatment of the signals involved in 2DES has been reported previously in the literature. ${ }^{24-25}$ Here we briefly introduce the main signals relevant for the modeling of the 2DES maps presented in the main manuscript. In our simulations, we thus start by calculating the total polarization in the time domain as $P=N\langle\hat{\mu}\rangle=N \operatorname{Tr}(\hat{\mu} \rho)$, with $N$ being the particle density and $\rho$ denoting the density matrix of the system. Hence, at each time delay $\tau, T$, we compute the time evolution of the density matrix along the simulation time $t$, by numerically solving the master equation in the Lindblad form ${ }^{26}$

$$
\dot{\rho}=-\frac{i}{\hbar}[H, \rho]+\sum_{n} \mathcal{L}_{n}(\rho)
$$


Here, the total Hamiltonian $H=H_{0}+H_{I}$ describes the free evolution of the system $H_{0}$ and its interaction with light $H_{I}$ (eqs 12-13). Dissipation processes, such as electronic dephasing and population relaxation, are accounted for phenomenologically within the Lindblad formalism. ${ }^{26}$ Specifically, they are described by superoperators $\mathcal{L}_{n}(\rho)$ of the form $\mathcal{L}_{n}(\rho)=\gamma_{n}\left(V_{n} \rho V_{n}^{\dagger}-\frac{1}{2}\left\{V_{n}^{\dagger} V_{n}, \rho\right\}\right)$, with $\gamma_{n}$ denoting the damping rate, and $V_{n}^{\dagger}$ and $V_{n}$ being the creation and annihilation operators for the $n$-th damping process. Note that, with the exception of the system Hamiltonian $H_{0}$, all other operators in the master equation (eq 17) depend on $(\tau, T, t)$.

For the $k$-th TLS in the system, the creation and annihilation operators describing electronic dephasing read $V_{\text {deph }}^{\dagger}=V_{\text {deph }}=\left|1_{k}\right\rangle\left\langle 1_{k}\right|$, whereas the ones for population relaxation to the ground state are taken as $V_{r e l}^{\dagger}=\left|1_{k}\right\rangle\left\langle 0_{k}\right|$ and $V_{r e l}=\left|0_{k}\right\rangle\left\langle 1_{k}\right|$, respectively. ${ }^{4}$

In both $\mathrm{X}$ and $\mathrm{C}$ models introduced in section 3.3.1, we take the electronic dephasing time as $T_{2}=30 \mathrm{fs}$. This dephasing time is estimated from the decay of the perturbed free-induction decay in the differential reflectivity (Figure 1b, negative time delays). The population relaxation time to the ground state is set to $T_{1}=100 \mathrm{ps}$, to mimic the decay dynamics at positive time delays in our experimental data (Figure 1d).

In the $\mathrm{C}$ model, we also include population relaxation within the free carrier continuum through an additional Lindblad superoperator, in which the corresponding relaxation operators $\left(V_{r e, C C}^{\dagger}\right.$ and $\left.V_{r e l, C C}\right)$ bring population from the higher to the next lower excited state along the chain of TLS with a rate $\gamma_{C C}=1 / T_{1, C C}$. In contrast to the operators for relaxation to the ground state ( $\left.V_{r e l}^{\dagger}, V_{r e l}\right)$ defined above, the relaxation operators $V_{r e l, C C}^{\dagger}, V_{r e l, C C}$ act on the excited states of neigh- 
boring TLS and not between excited and ground state of the same TLS. Specifically, the population relaxation from the $k$-th to the (k-1)-th TLS is described by the creation and annihilation operators $V_{\text {rel,CC }}^{\dagger}=\left|1_{k}\right\rangle\left\langle 1_{k-1}\right|$ and $V_{r e l, C C}=\left|1_{k-1}\right\rangle\left\langle 1_{k}\right|$. To match the observed 2DES spectra and dynamics at excitation into the free carrier continuum $(>2.45 \mathrm{eV})$ we need to account for a fast decay time of only $T_{1, C C}=15 \mathrm{fs}$.

We numerically solve eq 17 by a nonperturbative approach to yield $\rho(\tau, T, t)$, and then calculate the total polarization $P(\tau, T, t)=N \operatorname{Tr}\{\hat{\mu} \rho(\tau, T, t)\} \cdot{ }^{5}$ This polarization includes all allowed phase-matched signals as well as all linear and third-order nonlinear contributions arising from interaction with the pulse sequence. ${ }^{27}$ To isolate the nonlinear signals under phase-matched conditions as measured in our experiment, i.e., emitted in the probe direction, we apply a phasecycling algorithm. To this aim, at each time delay $\tau, T$, we calculate the total polarization for four different phases $\phi=n \pi / 2$, with $n=0,1,2,3$, between the pump-pair and the probe pulses and average them. The relative phase between pump and probe pulses is cycled by varying the absolute phase of each of the two pump pulses in steps of $\pi / 2$. The summation thus cancels out the polarization induced by the pump pulses and also all interferences between the pumpinduced fields and the probe fields. The resulting total polarization signal is therefore proportional to that probed experimentally. Subsequently, we subtract the linear contributions to the polarization induced by the interaction with the probe pulse alone. The phase-cycling scheme yields the directional dependence of the nonlinear, third order $P^{(3)}(\tau, T, t)$ signal as in our experiment and it is analogous to the one previously reported for pump-probe signals. ${ }^{28}$ Similar phase-cycling procedures have been reported for multidimensional spectroscopy in the literature by different groups. ${ }^{27,29-30}$ To obtain the 2DES reflection signals from the total polarization calculated in the time domain, we proceed as introduced in section 3.1. A Fourier transform along $t$ of the calculated total polarization yields the polarization spectrum $P(\tau, T, \omega)$. From 
this quantity, we extract the optical susceptibility $\chi(\tau, T, \omega) \approx \frac{P(\tau, T, \omega)}{E_{0}(\omega)}$ in the frequency domain. The reflectivity signal $R(\tau, T, \omega)$ is then approximated using the Fresnel equations for the reflection coefficient with the refractive index $n(\omega)=\sqrt{\varepsilon(\omega)} \approx \sqrt{\varepsilon_{\infty}+\chi(\omega)}$. Here the dielectric function of the sample consists of a non-resonant background $\varepsilon_{\infty}$, which we take as a constant to fit with the experiments, and a resonant part given by the calculated susceptibility $\chi$. Finally, at each waiting time $T$ we perform a Fourier transform along $\tau$ to obtain the 2DES energy-energy maps for excitation $E_{X}$ and detection $E_{D}$ energy, respectively. 


\section{Supplementary Figures}

(a)

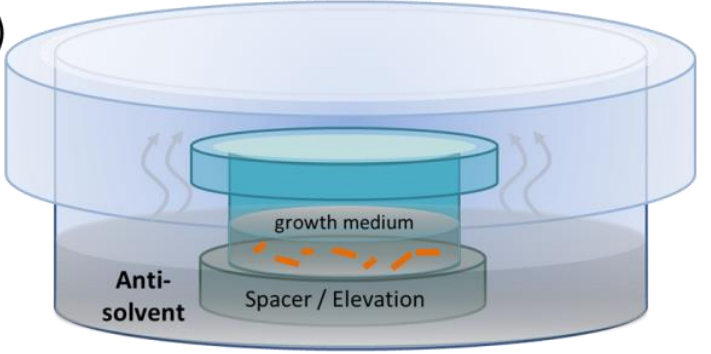

(b)

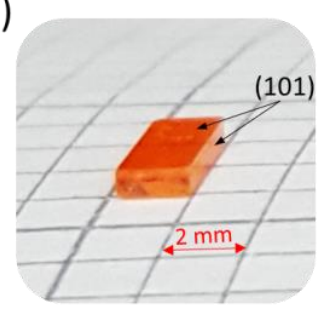

(c)

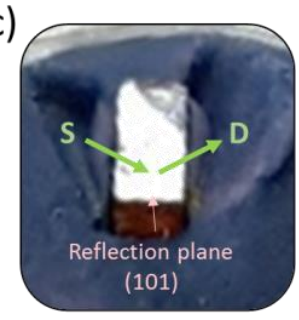

Figure S1: (a) Illustration of the crystal-growth setup. A filtered precursor solution is placed in a clean crystallization flask and covered with a filter paper and a glass petri dish on top to reduce anti-solvent vapor diffusion rate into the growth medium. The covered crystallization flask was then placed inside a deeper, flat-bottomed, glass dish, which contained the anti-solvent. Different architectures are possible, but a wide and flat crystallization flask has practical advantages. (b) A typical single crystal collected from the crystallization solution and dried with a soft tissue under $\mathrm{N}_{2}$ flow. (c) The crystallographic orientation is verified via XRD analysis in a specular reflection mode (between source (S) and detector (D)), where the (101) plane was set as the reflection plane. Further details can be found in Ref. 1.

(a)

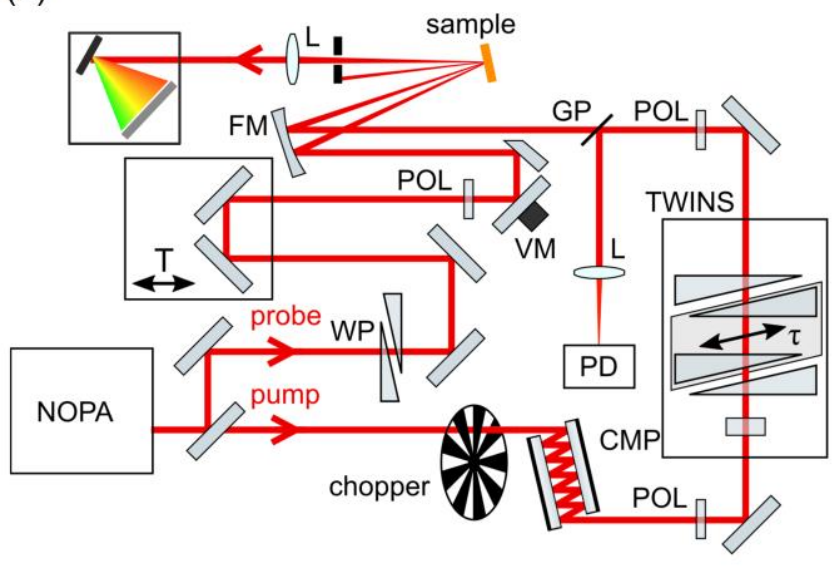

(b)

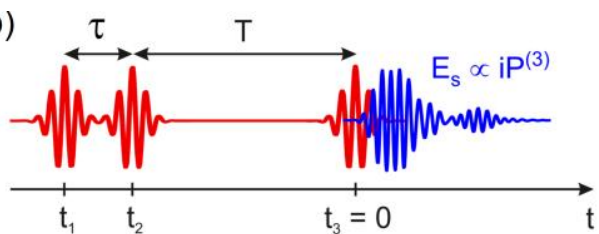

(c)

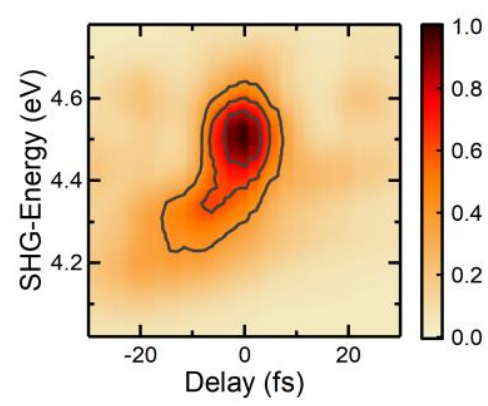

Figure S2: (a) Scheme of the home-built experimental setup used for pump-probe and twodimensional electronic spectroscopy (2DES). (b) Scheme of the pulse sequence interacting with the sample in a 2DES experiment. The time delay between the two pump pulses defines the coherence time $\tau$, whereas that between the second pump and the probe pulse is the waiting time $T$. (c) SHG-FROG map of the cross-correlation between pump and probe beams at the sample position, giving a time resolution of our experiment of $<13 \mathrm{fs}$. 


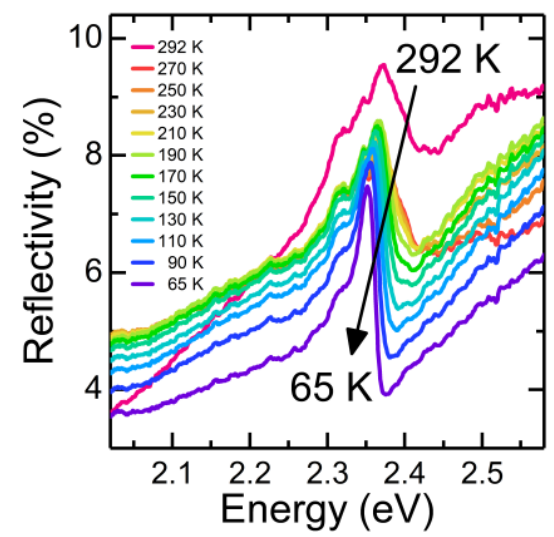

Figure S3: Temperature dependent linear reflectivity spectra of $\mathrm{Cs}_{\mathrm{PbBr}}$ single crystals show essentially a single dispersive resonance near the band-edge. At room temperature the resonance position is at $\sim 2.4 \mathrm{eV}$, in agreement with previous reports in the literature. ${ }^{8} \mathrm{~A}$ monotonic decrease of the resonance energy and a spectral linewidth narrowing are observed with decreasing temperature.

(a)

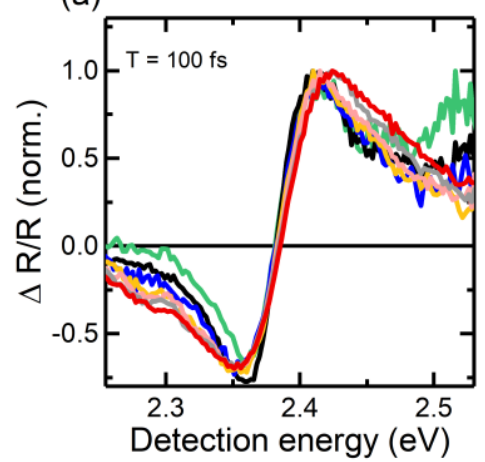

(c)

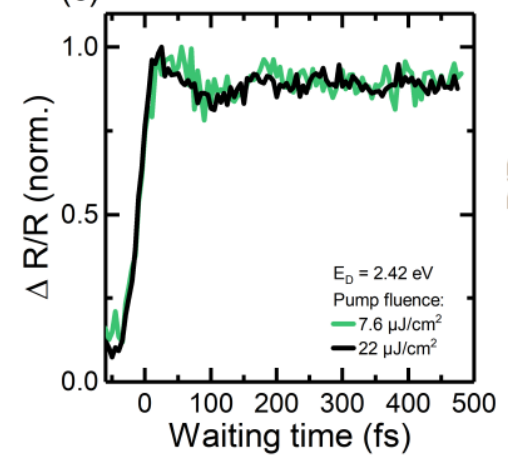

(b)

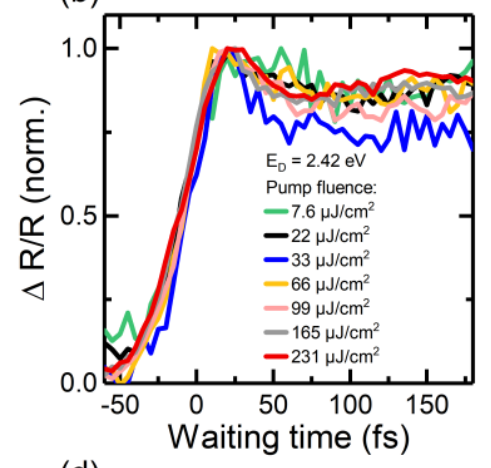

(d)

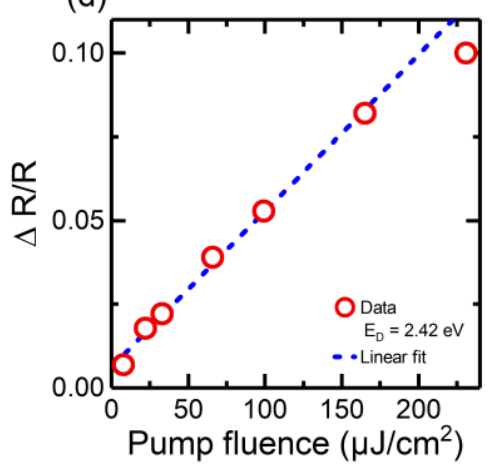

Figure S4: Pump-energy dependence of the differential reflectivity $\Delta R / R$. The pump fluence is varied between $\sim 8$ and $\sim 230 \mu \mathrm{J} / \mathrm{cm}^{2}$. (a) The normalized spectra at a time delay of $T=100 \mathrm{fs}$ show no significant variation in the shape of the $\Delta R / R$ spectra over the entire investigated pump-energy range. (b, c) Also, the early time decay dynamics at a probe energy of $E_{D}=2.42$ $\mathrm{eV}$ remain essentially unchanged with increasing photoexcited carrier density. (d) Differential reflectivity $\Delta R / R$ signal averaged over waiting times between 125 and 175 fs (red open circles) in (b), showing a linear increase in signal amplitude (fit, blue dashed line) with the excitation fluence up to $\sim 170 \mu \mathrm{J} / \mathrm{cm}^{2}$. For the highest pump fluence investigated here, a slight deviation from a linear dependence is observed, an indication of approaching the Mott density. 
(a)

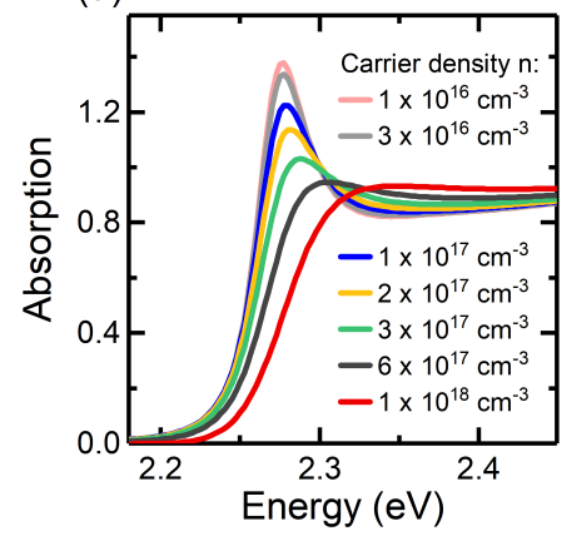

(b)

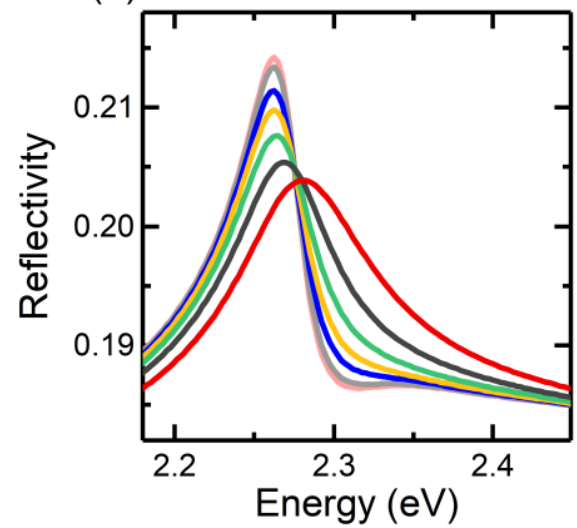

Figure S5: Excitation-energy dependence of the calculated linear (a) absorption and (b) reflectivity spectra at room temperature including many-body effects. A pronounced reduction of the excitonic contribution to the spectra with increasing excitation density results in an apparent blue shift of the spectrum. For excitation densities of about $1 \cdot 10^{18} \mathrm{~cm}^{-3}$ (red), the Mott transition is reached and the spectra reflect essentially only the contribution of the carrier continuum.

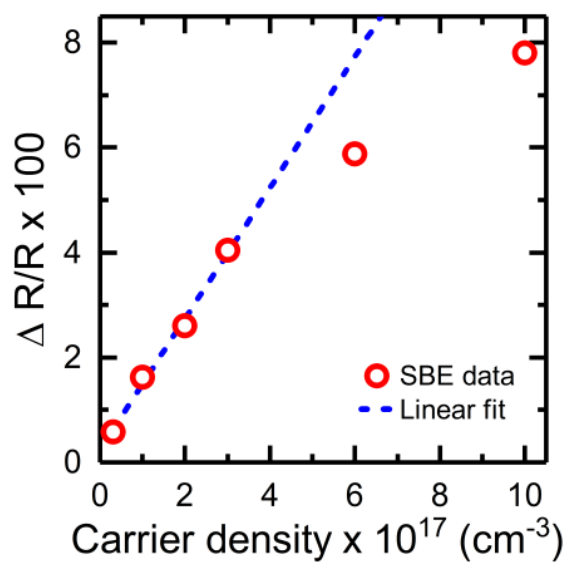

Figure S6. Maximum signal amplitude of the differential reflectivity $\Delta R / R$, calculated using the semiconductor Bloch equations and shown in Figure 1e of the manuscript, as a function of the excited carrier density. The simulations show a linear increase of $\Delta R / R$ up to carrier densities of $3 \cdot 10^{17} \mathrm{~cm}^{-3}$ and indicate a Mott density of $10^{18} \mathrm{~cm}^{-3}$. 


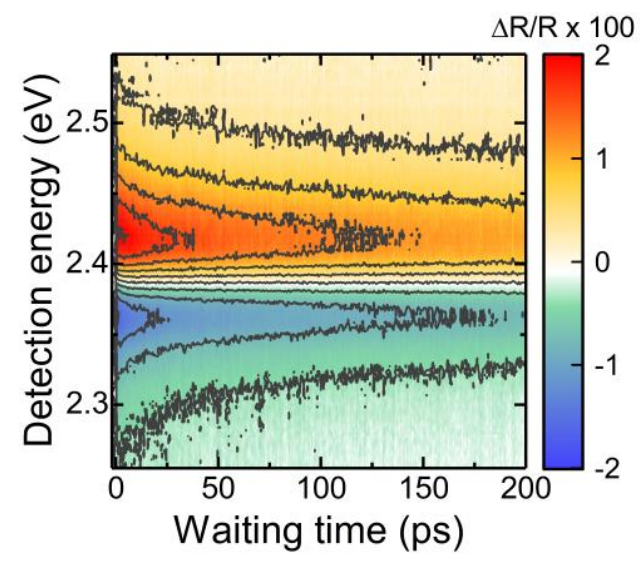

Figure S7: Differential reflectivity $\Delta R / R$ map of $\mathrm{CsPbBr}_{3}$ single crystals for waiting times of up to $200 \mathrm{ps}$. The map shows essentially a single dispersive resonance at $\sim 2.4 \mathrm{eV}$. The amplitude of this peak decays biexponentially with time constants of $\sim 10$ ps and $\sim 700$ ps.
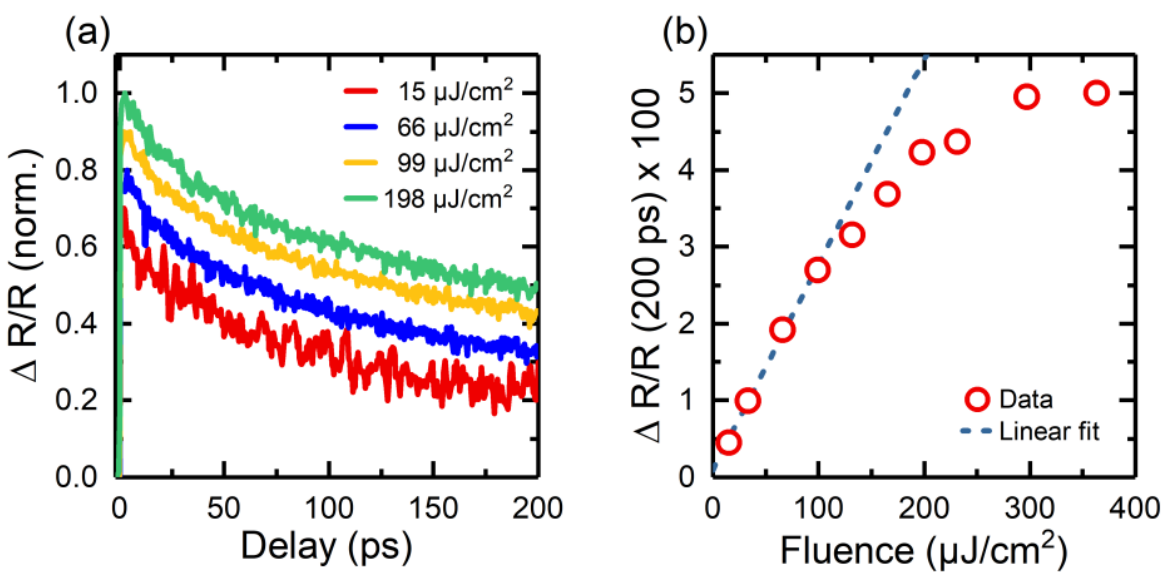

Figure S8. (a) Pump-fluence dependence of the differential reflectivity $\Delta R / R$ dynamics recorded at a probe energy of $2.42 \mathrm{eV}$. The curves are normalized to the maximum and vertically shifted by 0.1 for clarity. No significant variations of the decay dynamics are observed. (b) Amplitude of the $\Delta R / R$ signal (red circles) at a delay of $200 \mathrm{ps}$ as a function of the pump fluence. All data in (a) for fluences of $<100 \mu \mathrm{J} / \mathrm{cm}^{2}$ show a linear dependence (blue dashed) on the pump fluence. 
(a)

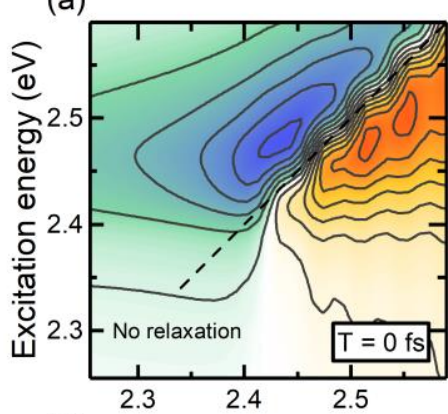

(c)

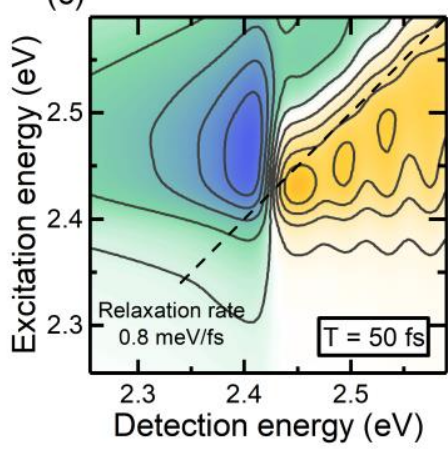

(b)

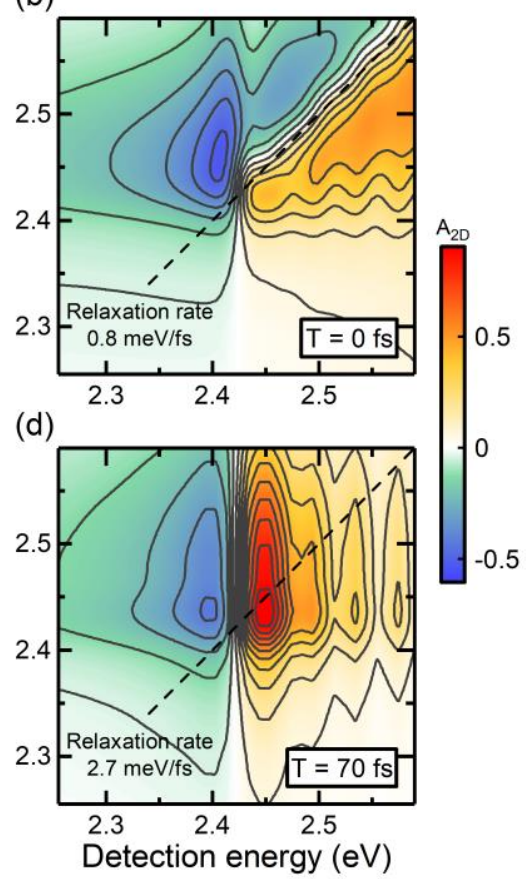

Figure S9: Model simulations of 2DES maps performed under the assumption that only free carrier transitions contribute to the optical spectrum. Free carriers in the valence and conduction band are modeled as a system of uncoupled two-level systems with transition dipole moments following the Elliot model for the free carrier continuum. The simulations are performed (a) in the absence of relaxation to the band-edge and (b-c) with a relaxation rate of $0.8 \mathrm{meV} / \mathrm{fs}$ and (d) $2.7 \mathrm{meV} / \mathrm{fs}$. Dispersive lineshapes along the detection energy axis arise from the simulation of the reflection signals.

(a)

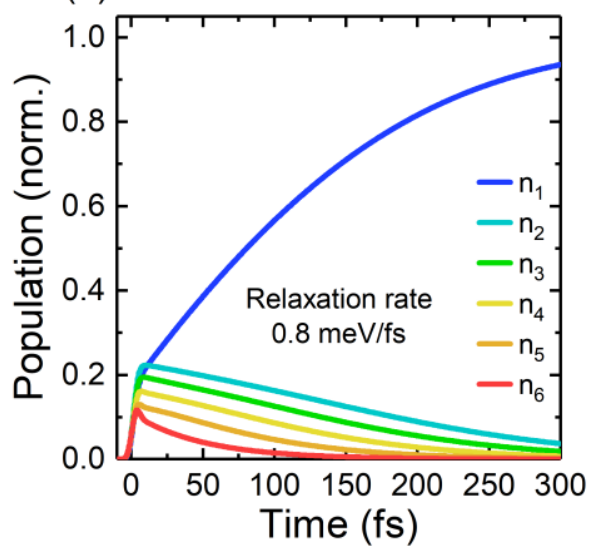

(b)

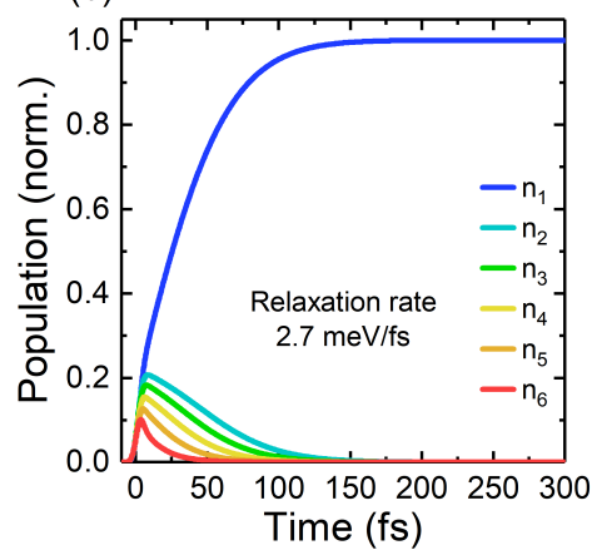

Figure S10: Model simulations of the free carrier relaxation for a relaxation rate of (a) 0.8 $\mathrm{meV} / \mathrm{fs}$ and (b) $2.7 \mathrm{meV} / \mathrm{fs}$. The free carrier population is created by a 6 -fs pump pulse at $0 \mathrm{fs}$. The excess charge density $n_{i}$ in the $i$-th excited state is color-coded. 

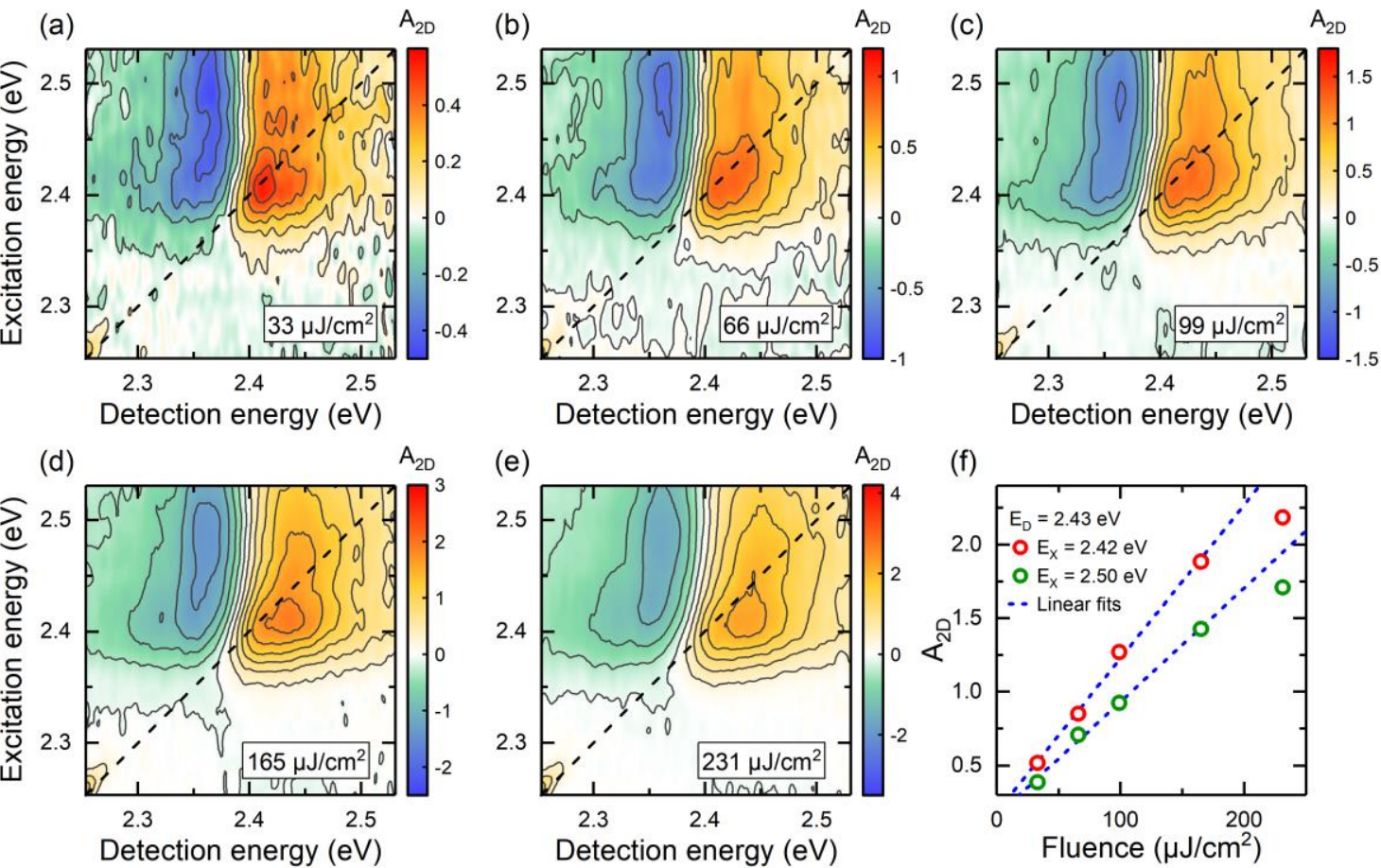

Figure S11. (a-e) 2DES spectra of a $\mathrm{CsPbBr}_{3}$ crystal recorded at room temperature and at a fixed waiting time of $70 \mathrm{fs}$, varying the pump fluence between (a) 33 and (e) $231 \mu \mathrm{J} / \mathrm{cm}^{2}$. Within this range, the variation of the pump fluence has no significant effect on the shape of the 2DES spectra. (f) Amplitude of the 2DES signal at excitation energies of $2.42 \mathrm{eV}$ (red circles) and $2.50 \mathrm{eV}$ (green circles) and a detection energy of $2.43 \mathrm{eV}$ as a function of the pump fluence. The dashed blue lines are linear fits to the data.

\section{References}

1. Rakita, Y.; Kedem, N.; Gupta, S.; Sadhanala, A.; Kalchenko, V.; Böhm, M. L.; Kulbak, M.; Friend, R. H.; Cahen, D.; Hodes, G., Low-Temperature Solution-Grown CsPbBr3 Single Crystals and Their Characterization. Cryst. Growth Des 2016, 16, 5717-5725.

2. Brida, D.; Manzoni, C.; Cerullo, G., Phase-Locked Pulses for Two-Dimensional Spectroscopy by a Birefringent Delay Line. Opt. Lett. 2012, 37, 3027-3029.

3. Réhault, J.; Maiuri, M.; Oriana, A.; Cerullo, G., Two-Dimensional Electronic Spectroscopy with Birefringent Wedges. Rev. Sci. Instrum. 2014, 85, 123107.

4. De Sio, A.; Troiani, F.; Maiuri, M.; Réhault, J.; Sommer, E.; Lim, J.; Huelga, S. F.; Plenio, M. B.; Rozzi, C. A.; Cerullo, G.; Molinari, E.; Lienau, C., Tracking the Coherent Generation of Polaron Pairs in Conjugated Polymers. Nat. Commun. 2016, 7, 13742.

5. De Sio, A.; Lienau, C., Vibronic Coupling in Organic Semiconductors for Photovoltaics. Phys. Chem. Chem. Phys. 2017, 19, 18813-18830.

6. Li, H.; Moody, G.; Cundiff, S. T., Reflection Optical Two-Dimensional Fourier-Transform Spectroscopy. Opt. Express 2013, 21, 1687-1692.

7. Trebino, R.; DeLong, K. W.; Fittinghoff, D. N.; Sweetser, J. N.; Krumbügel, M. A.; Richman, B. A.; Kane, D. J., Measuring Ultrashort Laser Pulses in the Time-Frequency Domain Using FrequencyResolved Optical Gating. Rev. Sci. Instrum. 1997, 68, 3277-3295.

8. Sebastian, M.; Peters, J. A.; Stoumpos, C. C.; Im, J.; Kostina, S. S.; Liu, Z.; Kanatzidis, M. G.; Freeman, A. J.; Wessels, B. W., Excitonic Emissions and above-Band-Gap Luminescence in the SingleCrystal Perovskite Semiconductors $\mathrm{CsPbBr} 3$ and CsPbCl3. Phys. Rev. B 2015, 92. 
9. $\quad$ Yang, Z.; Surrente, A.; Galkowski, K.; Miyata, A.; Portugall, O.; Sutton, R. J.; Haghighirad, A. A.; Snaith, H. J.; Maude, D. K.; Plochocka, P.; Nicholas, R. J., Impact of the Halide Cage on the Electronic Properties of Fully Inorganic Cesium Lead Halide Perovskites. ACS Energy Lett. 2017, 2, 1621-1627.

10. Monahan, D. M.; Guo, L.; Lin, J.; Dou, L.; Yang, P.; Fleming, G. R., Room-Temperature Coherent Optical Phonon in 2D Electronic Spectra of CH3NH3Pbl3 Perovskite as a Possible Cooling Bottleneck. J. Phys. Chem. Lett. 2017, 8, 3211-3215.

11. Jha, A.; Duan, H.-G.; Tiwari, V.; Nayak, P. K.; Snaith, H. J.; Thorwart, M.; Miller, R. J. D., Direct Observation of Ultrafast Exciton Dissociation in Lead lodide Perovskite by 2D Electronic Spectroscopy. ACS Photonics 2018, 5, 852-860.

12. Richter, J. M.; Branchi, F.; Valduga de Almeida Camargo, F.; Zhao, B.; Friend, R. H.; Cerullo, G.; Deschler, F., Ultrafast Carrier Thermalization in Lead lodide Perovskite Probed with Two-Dimensional Electronic Spectroscopy. Nat. Commun. 2017, 8, 376.

13. Haug, H.; Koch, S. W., Quantum Theory of the Optical and Electronic Properties of Semiconductors. 5th ed.; World Scientific: Singapore ; Hackensack, N.J., 2009; $p$ xiii, 469 pages.

14. Jahnke, F.; Kira, M.; Koch, S. W., Linear and Nonlinear Optical Properties of Excitons in Semiconductor Quantum Wells and Microcavities. Z. Phys. B Con. Mat. 1997, 104, 559-572.

15. Schäfer, W.; Wegener, M., Semiconductor Optics and Transport Phenomena. Springer Berlin Heidelberg: 2013.

16. Manzke, G.; Klähn, T.; Henneberger, K., Quantum Kinetics and Linear Optical Response of Semiconductors. Phys. Status Solidi C 2003, 0, 1480-1483.

17. Strinati, G., Effects of Dynamical Screening on Resonances at Inner-Shell Thresholds in Semiconductors. Phys. Rev. B 1984, 29, 5718-5726.

18. Bornath, T.; Kremp, D.; Schlanges, M., Two-Particle Problem in a Nonequilibrium Many-Particle System. Phys. Rev. E 1999, 60, 6382-6394.

19. Kang, Y.; Han, S., Intrinsic Carrier Mobility of Cesium Lead Halide Perovskites. Phys. Rev. Appl. 2018, 10, 044013.

20. Becker, M. A.; Vaxenburg, R.; Nedelcu, G.; Sercel, P. C.; Shabaev, A.; Mehl, M. J.; Michopoulos, J. G.; Lambrakos, S. G.; Bernstein, N.; Lyons, J. L.; Stoferle, T.; Mahrt, R. F.; Kovalenko, M. V.; Norris, D. J.; Raino, G.; Efros, A. L., Bright Triplet Excitons in Caesium Lead Halide Perovskites. Nature 2018, 553, 189-193.

21. Scully, M. O.; Zubairy, M. S., Quantum Optics. Cambridge University Press: Cambridge ; New York, 1997; p xxi, 630 p.

22. Haroche, S.; Raimond, J. M., Exploring the Quantum: Atoms, Cavities, and Photons. OUP Oxford: 2013.

23. Elliott, R. J., Intensity of Optical Absorption by Excitons. Phys. Rev. 1957, 108, 1384-1389.

24. Jonas, D. M., Two-Dimensional Femtosecond Spectroscopy. Annu. Rev. Phys. Chem. 2003, 54, 425-463.

25. Mukamel, S., Multidimensional Femtosecond Correlation Spectroscopies of Electronic and Vibrational Excitations. Annu. Rev. Phys. Chem. 2000, 51, 691-729.

26. Palmieri, B.; Abramavicius, D.; Mukamel, S., Lindblad Equations for Strongly Coupled Populations and Coherences in Photosynthetic Complexes. J. Chem. Phys. 2009, 130, 204512.

27. Gelin, M. F.; Egorova, D.; Domcke, W., Efficient Calculation of Time- and Frequency-Resolved Four-Wave-Mixing Signals. Acc. Chem. Res. 2009, 42, 1290-1298.

28. Seidner, L.; Stock, G.; Domcke, W., Nonperturbative Approach to Femtosecond Spectroscopy: General Theory and Application to Multidimensional Nonadiabatic Photoisomerization Processes. J. Chem. Phys. 1995, 103, 3998-4011.

29. Meyer, S.; Engel, V., Non-Perturbative Wave-Packet Calculations of Time-Resolved Four-WaveMixing Signals. Appl. Phys. B 2000, 71, 293-297.

30. Mančal, T.; Pisliakov, A. V.; Fleming, G. R., Two-Dimensional Optical Three-Pulse Photon Echo Spectroscopy. I. Nonperturbative Approach to the Calculation of Spectra. J. Chem. Phys. 2006, 124, 234504. 